\title{
A new shell element taking thickness-stretchability into account for mechanics-based springback compensation system
}

\author{
Hibiki Arashiyama ${ }^{1}$, Tetsuo Oya ${ }^{1}$ and Koichi Ito ${ }^{2}$ \\ ${ }^{1}$ Graduate School of Science and Technology, Keio University, 14109 Kouhoku-ku, Yokohama-shi, Japan \\ ${ }^{2} M \& M$ Reserch Ltd, 13113 Shibuya-ku, Japan
}

\begin{abstract}
To reduce springback, the coining process is often applied in sheet metal forming. However, the finite element method with a conventional shell element cannot formulate this process because of the plane stress condition. Therefore, in this study, a new shell element, which has extra nodes called pseudo-nodes, was developed to calculate the through-thickness stress. Using this element, a mechanics-based springback compensation system was constructed and tested.
\end{abstract}

\section{Introduction}

Springback is a serious problem in sheet metal forming. It has become increasingly important to solve this problem because of the use of AHSS (advanced high-strength steel), which exhibits a large amount of springback, particularly in the automobile industry. Springback is the elastic deformation driven by residual stress generated during the forming process. Thus, the bending moment before springback is the main factor causing springback. To solve the problem of springback, several tool compensation methods have been developed. Gan and Wagoner proposed the displacement adjustment (DA) method, which is the most effective means of compensating for the springback in tools [1], and Karafillis and Boyce developed the force descriptor method (FDM) [2][3]. Recently, various methods based on the DA method or FDM have been reported such as the comprehensive compensation method [4], the accelerated compensation method [5], and the smooth DA method [6], and so forth. However, the cause of springback, i.e., the bending moment before springback, is not considered in these methods. In the DA method, the compensated shape can be obtained using only geometric data. FDM has no ground for the variation of springforward obtained by reversing the nodal force at the end of the stamping process. Therefore, these methods are not based on springback mechanics. Thus, there is a high probability that the shape does not converge to the compensated shape. In addition, it takes considerable time to determine the compensated shape because their algorithms involve forming analysis. Moreover, in the conventional methods, although the shape can be obtained, other working parameters such as the sheet normal pressure and in-plane stretching force cannot be obtained. Thus, tool design cannot be conducted from the viewpoint of the designer.

Therefore, in our study, a new mechanics-based springback compensation system was developed. This system can take account of mechanical relationships account into springback compensation, by calculating the bending moment derived from not only the curvature but also the pressure and tension.

The finite element method with a conventional shell element cannot formulate the coining process because of the plane stress condition. By employing a solid element instead of a shell element, the normal stress can be calculated but it takes considerable time. Thus, we propose a new shell element, which has extra nodes called pseudo-nodes, to calculate the normal stress. In this study, using this element, a mechanics-based springback compensation system was constructed and tested.

\section{Concept of springback compensation system}

\subsection{Springback mechanics [7]}

Springback is an elastic deformation caused by residual stress generated at the end of the forming process. Thus, the bending moment before springback (at the end of forming analysis) is the main cause of springback. This means that the shape after springback is determined by the conditions at the end of forming analysis. Springback is calculated by the following mechanical equation:

$$
\boldsymbol{D}\left(\boldsymbol{\kappa}_{\text {after }}-\boldsymbol{\kappa}_{\text {before }}\right)=-\boldsymbol{M}_{\text {before }},
$$

\footnotetext{
a Hibiki Arashiyama: arashiyamah@a6.keio.jp
} 
where $\boldsymbol{\kappa}_{\text {after }}, \boldsymbol{\kappa}_{\text {before }}, \boldsymbol{M}_{\text {before }}$ and $\boldsymbol{D}$ are the curvature after springback, curvature before springback, bending moment before springback, and elastic bending rigidity, respectively. This equation is called the springback mechanical equation in this paper. To calculate $\boldsymbol{\kappa}_{\text {before }}$ and $\boldsymbol{M}_{\text {before, }}$ information such as the nodal coordinates and the stress tensor at the end of forming is essential.

\subsection{Concept of springback compensation}

Springback is expressed by eq.(1). By substituting $\boldsymbol{\kappa}_{\text {desired }}$, the curvature of the desired shape, for $\boldsymbol{\kappa}_{\text {after }}$ in eq.(1), $\boldsymbol{\kappa}_{\text {before }}$ and $\boldsymbol{M}_{\text {before }}$ for the desired shape are obtained. This relationship is expressed as

$$
\boldsymbol{D}\left(\boldsymbol{\kappa}_{\text {desired }}-\boldsymbol{\kappa}_{\text {before }}\right)=-\boldsymbol{M}_{\text {desired }}\left(\boldsymbol{\kappa}_{\text {before }}, \boldsymbol{P}\right),
$$

where $\boldsymbol{P}$ and $\boldsymbol{T}$ is a force vectors describing the sheet normal pressure and the in-plane stretching force, respectively. Also, $\boldsymbol{M}_{\text {before }}$ is defined as a function of $\boldsymbol{\kappa}_{\text {before, }} \boldsymbol{P}$ and $\boldsymbol{T}$. To determine the conditions before springback, it is necessary that eq.(2) is satisfied. However, eq.(2) cannot be solved if a target has a complex structure. Therefore, $\boldsymbol{M}_{\text {before }}$ is determined by users by repeatedly adjusting the parameters $\boldsymbol{\kappa}_{\text {before }}$ and $\boldsymbol{P}$ and $\boldsymbol{T}$. Figures 1 and 2 respectively show outlines of the conventional springback compensation method and the proposed method. Firstly, forming analysis and springback analysis are performed, then it is examined whether the shape is within the specified tolerance. If necessary, the bending moment before springback is modified by updating only the shape (curvature) in the conventional system. However in the proposed system, it is modified by updating the curvature distribution and the force vector in the subroutine.

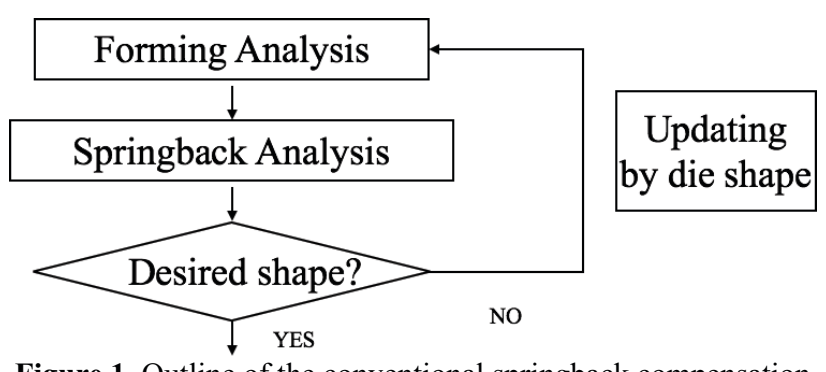

Figure 1. Outline of the conventional springback compensation system.

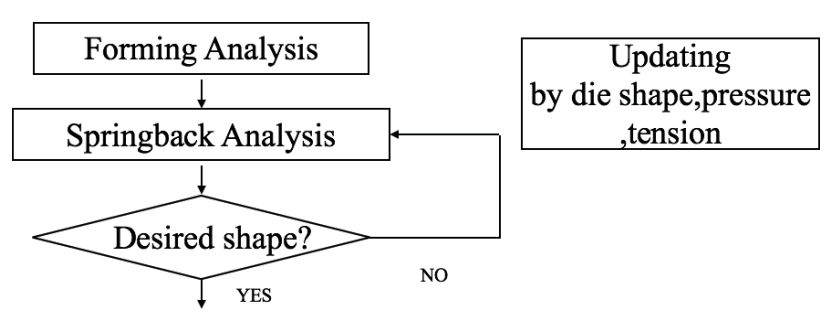

Figure 2. Outline of the proposed springback compensation system.

\section{Formulation of the shell element}

\subsection{Outline}

As mentioned above, it is necessary in springback compensation to predict the bending moment concisely in the subroutine of the proposed system. For this purpose, a new shell element is developed. The features of the proposed shell element are as follows.

a) The sheet-normal strain rate is introduced using an enhanced strain formulation.

b) A Fully three-dimensional constitutive relationship is used.

c) The formulation is completed by adding only the terms associated with the enhanced strain to the expressions for the conventional iso-parametric four-nodes shell element.

The enhanced strain formulation is a novel idea used to treat the normal stress for a shell element. When the shell element is applied to a sheet metal forming simulation, it is expected that the effect of the sheet normal pressure on the bending moment can be evaluated, furthermore, the constraint on both sides of the sheet (e.g. constraints by punch and the die) can be treated.

\subsection{Formulation of the shell element}

The sheet-normal strain rate, which is not considered in a conventional shell elements based on the MindlinReissner's assumption, is introduced as an enhanced strain component in the iso-parametric four-nodes shell element. Assuming that the introduced strain rate varies linearly in the sheet thickness direction and is uniform over the sheet surface, the expression for the sheetnormal strain rate $\varepsilon$ is

$$
\varepsilon=\frac{2\left(\overline{v_{U}}+\overline{v_{L}}\right)}{h} \xi_{3}+\frac{\overline{v_{U}}-\overline{v_{L}}}{h},
$$

where $\xi_{3}, h$, and $\left(\bar{v}_{U}, \bar{v}_{L}\right)$ are the natural coordinate in the sheet normal direction, the sheet thickness, and the relative velocities at strain evaluation points $U$ and $L$ from the midsurface, respectively (see Figure.3). The relative velocities $\overline{\boldsymbol{v}}_{\boldsymbol{U}}$ and $\bar{v}_{\boldsymbol{L}}$ are defined as velocities consistent with the enhanced strain component and are independently of the nodal velocities for a conventional shell element.

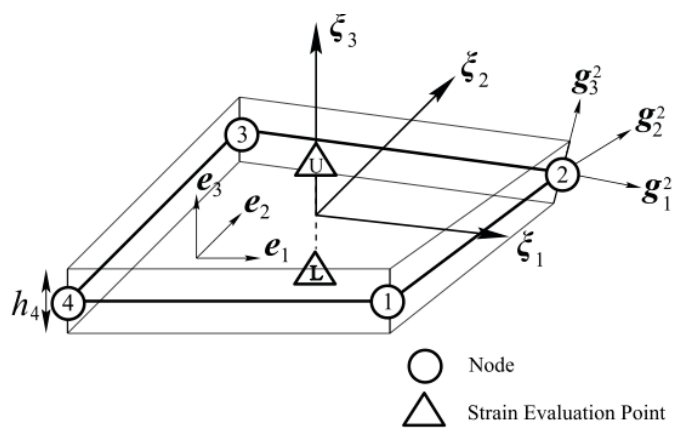

Figure 3. Configuration of the new element. 


\subsection{Velocity field consistent with the enhanced strain}

Assuming $\varepsilon=\partial v / \partial\left(2 h \xi_{3}\right)$ and integrating eq.(3) with respect to $h \xi_{3}$, we obtain the velocity field $v\left(\xi_{3}\right)$ consistent with the enhanced strain rate as

$$
v\left(\xi_{3}\right)=\frac{\overline{v_{U}}+\overline{v_{L}}}{h} \xi_{3}^{2}+\frac{\overline{v_{U}}-\overline{v_{L}}}{h} \xi_{3} .
$$

Then, we assume that the sheet normal velocity at the midsurface $v_{M}$ is defined by the mean value of the sheetnormal nodal velocity,

$$
v_{M}=\frac{1}{4} \sum_{\alpha=1}^{4} v_{3}^{\alpha}
$$

where $v_{3}^{\alpha},(\alpha=1,2,3,4)$ are the sheet normal nodal velocity components. Denoting $v_{U}$, and $v_{\boldsymbol{L}}$ as the consistent velocities at the strain evaluation points and setting the strain evaluation points $U$ and $L$ at the center of the shell on the upper and lower surfaces, respectively, we obtain

$$
\overline{v_{U}}=v_{U}-v_{M}, \overline{v_{L}}=v_{L}-v_{M} .
$$

Substituting eqs.(5) and (6) into eq.(4), we obtain the following expression for the consistent velocity field in the sheet thickness direction:

$$
v\left(\xi_{3}\right)=-\frac{\xi_{3}^{2}}{4} \sum_{\alpha=1}^{4} v_{3}^{\alpha}+\frac{\xi_{3}\left(\xi_{3}-1\right)}{2} v_{L}+\frac{\xi_{3}\left(\xi_{3}+1\right)}{2} v_{U} .
$$

\subsection{Velocity interpolation for the new shell element}

The velocity field consistent with the enhanced strain $v\left(\xi_{3}\right)$ corresponds to the sheet-normal velocity field over the shell element. Therefore, we only need to add eq.(7) as the sheet normal velocity to the expression used for velocity interpolation for the conventional four-nodes isoparametric shell element. Let indices 1, 2, and 3 correspond to in-plane variables and sheet normal variables. Then we can express the velocity interpolation in terms of the nodal velocities $v_{i}^{\alpha}$ and the consistent velocities at the enhanced strain evaluation points $v_{L}$ and $v_{U}$ as

$$
v_{i}\left(\xi_{1}, \xi_{2}, \xi_{3}\right)=\sum_{\alpha=1}^{4} N^{\alpha}\left(\xi_{1}, \xi_{2}\right)\left[v_{i}^{\alpha}+\frac{1}{2} \xi_{3} h^{\alpha}\left(g_{1 i}^{\alpha} \dot{\theta}_{2}^{\alpha}-g_{2 i}^{\alpha} \dot{\theta}_{1}^{\alpha}\right)\right]
$$

$$
\begin{aligned}
v_{3}\left(\xi_{1}, \xi_{2}, \xi_{3}\right)= & \sum_{\alpha=1}^{4}\left[\left(N^{\alpha}\left(\xi_{1}, \xi_{2}\right)-\frac{\xi_{3}^{2}}{4}\right) v_{3}^{\alpha}\right. \\
& \left.+\frac{1}{2} N^{\alpha}\left(\xi_{1}, \xi_{2}\right) \xi_{3} h^{\alpha}\left(g_{13}^{\alpha} \dot{\theta}_{2}^{\alpha}-g_{23}^{\alpha} \dot{\theta}_{1}^{\alpha}\right)\right] \\
& +\frac{\xi_{3}\left(\xi_{3}-1\right)}{2} v_{L}+\frac{\xi_{3}\left(\xi_{3}+1\right)}{2} v_{U}
\end{aligned}
$$

where $N^{\alpha}\left(\xi_{1}, \xi_{2}\right), g_{i j}{ }^{\alpha}$ and $\dot{\theta}_{\boldsymbol{J}}{ }^{\alpha}$ are the conventional shape function for a four-nodes iso-parametric shell element, the $j$-th component of the base vector $\boldsymbol{g}^{\alpha}{ }_{i}$ of the nodal frame for node $\alpha$ (see. Figure 3), and the rotation angle around the $\boldsymbol{g}_{j}^{\alpha}$ axis, respectively. The new shell element has 22 degrees of freedom, which are composed of four nodal velocity vectors $v^{\alpha}=\left(v_{1}{ }^{\alpha}, v_{2}^{\alpha}, v_{3}^{\alpha}, \theta_{1}^{\alpha}, \theta_{2}^{\alpha}\right)^{T}$ and the strain consistent velocity vector $\boldsymbol{v}^{c}=\left(v_{L}, v_{U}\right)^{T}$. The relationships between the velocity field over the shell element and these 22 element variables are completely expressed in eq.(8). On the basis of this expression, it is possible to use the same method as in the case of the conventional shell element to obtain the element stiffness equation. To describe the case of no transverse shear under a uniform bending deformation, it is recommended to adopt the assumed strain formulation for transverse shear strain components.

\subsection{Contact and equivalent nodal forces}

To treat the constraints on both sides of the sheet constraints, i.e., those on the upper surface and lower surface, only the strain evaluation points are subjected to the sheet normal constraints, in other words, the four nodes of the shell are free from sheet-normal contact conditions. We consider that the shell element is subjected to external forces at points $U$ and $L$, as shown in Figure 4. The principle of the virtual work in relation to the external force and the equivalent nodal forces is expressed by

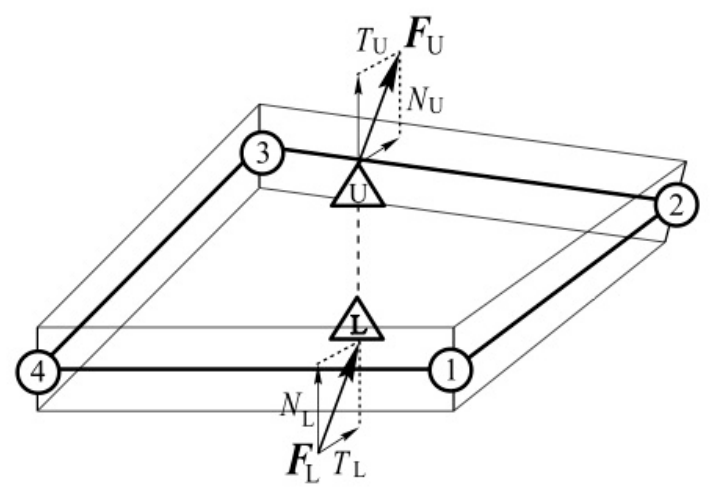

Figure 4. External forces applied to a shell element. 


$$
\begin{aligned}
\sum_{\alpha=1}^{4}(\delta \boldsymbol{v})^{T} \cdot \boldsymbol{f}+\delta v_{U} f_{U}+\delta v_{L} f_{L}=\int \delta \boldsymbol{v}\left(\xi_{1}, \xi_{2}, 1\right) \cdot \boldsymbol{F}_{U} d S_{U} \\
+\int \delta \boldsymbol{v}\left(\xi_{1}, \xi_{2},-1\right) \cdot \boldsymbol{F}_{L} d S_{L}
\end{aligned}
$$

where $\boldsymbol{f}_{\alpha}, \boldsymbol{f}_{U}$ and $\boldsymbol{f}_{L}$ are the equivalent nodal forces, and the equivalent forces at strain evaluation points $U$ and $L$, respectively. The first integration on the right-hand side of eq.(9) is performed over the upper surface and the second one is performed over the lower surface. Substituting eq.(8) into eq.(9) and assuming that $\boldsymbol{f}_{U}$ and $\boldsymbol{f}_{U}$ are the resultant forces in the case of a uniformly distributed surface load, some calculation leads to the following expressions.

$$
\begin{aligned}
& f_{i}^{\alpha}=T_{U i} \int N^{\alpha}\left(\xi_{1}, \xi_{2}\right) d S_{U}+T_{L i} \int N^{\alpha}\left(\xi_{1}, \xi_{2}\right) d S_{L} \quad(i=1,2) \\
& f_{\theta 1}^{\alpha}=\frac{1}{2} h^{\alpha}\left(g_{21}^{\alpha}+g_{22}^{\alpha}\right)\left[-T_{U 1} \int N^{\alpha}\left(\xi_{1}, \xi_{2}\right) d S_{U}+T_{L 1} \int N^{\alpha}\left(\xi_{1}, \xi_{2}\right) d S_{L}\right] \\
& f_{\theta 2}^{\alpha}=\frac{1}{2} h^{\alpha}\left(g_{11}^{\alpha}+g_{12}^{\alpha}\right)\left[T_{U 1} \int N^{\alpha}\left(\xi_{1}, \xi_{2}\right) d S_{U}-T_{L 1} \int N^{\alpha}\left(\xi_{1}, \xi_{2}\right) d S_{L}\right] \\
& f_{U}=N_{U} \\
& f_{L}=N_{L}
\end{aligned}
$$

In the new shell element, the sheet normal force, for example, the hydrostatic pressure or contact force, applies only to the two strain evaluation points, while the four nodes, which are located on the midsurface are subjected to an in-plane force, such as a friction force.

\section{Forming analysis with proposed element}

To show that the proposed element behaves in the desired manner, the forming analysis of a beam with constant curvature is demonstrated. Setting the forced rotational displacement to the endpoint of the beam, a flat sheet is bent so that $y$, the rotational freedom of the endpoint $\theta$, is $\pi / 6$ as shown in Figure 5. Table 1 shows the material and geometrical properties of the sheet. Springback analysis was performed using the developed solver and the shape after springback was compared with the theoretical value. Ten shell elements were used in this analysis. Figure 6 shows the results for the curvature radius obtained by the analysis. The curvature radius after springback obtained by the developed solver is close to the theoretical value. Therefore, the proposed element behaves in the desired manner in forming analysis.

Table 1. Material and geometrical properties.

\begin{tabular}{|c|c|c|c|c|}
\hline $\begin{array}{c}\text { Young's } \\
\text { modulus } \\
\text { /GPa }\end{array}$ & $\begin{array}{c}\text { Poisson's } \\
\text { ratio }\end{array}$ & $\begin{array}{c}\text { Length } \\
\text { /mm }\end{array}$ & $\begin{array}{c}\text { Width } \\
\text { /mm }\end{array}$ & $\begin{array}{c}\text { Thickness } \\
\text { /mm }\end{array}$ \\
\hline 210 & 0.3 & 100 & 10 & 1 \\
\hline
\end{tabular}

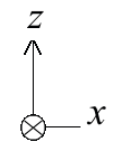

$y$

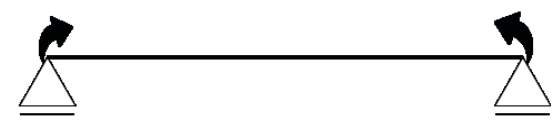

Figure 5. The schematic diagram of forming analysis.

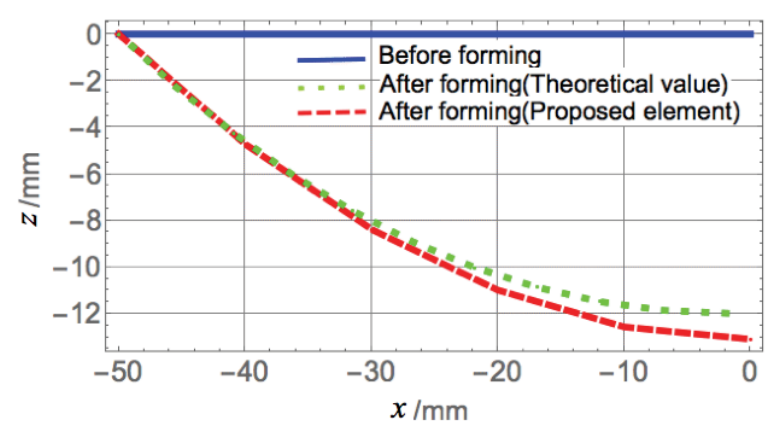

Figure 6. Results of forming analysis.

\section{Coining simulation and comparison with solid element}

To show that the proposed element behaves as desired in the coining process, a coining simulation is demonstrated. Figure 7 shows the FE model used in the coining simulation of bending. After carrying out forming analysis using LS-DYNA, coining analysis was carried out by applying pseudo-nodes to nodal forces. Table 2 shows the material properties of the sheet. Then, the coining simulation was performed using the solver and the radius of curvature after coining was compared with those when using a solid element and the conventional shell element. Ten shell elements were used. Figure 8 and Table 3 show the results for the radius of curvature obtained by the analysis. When springback is induced, the sheet unrolls from its original shape; therefore, the radius of curvature indicates the extent of springback. Although the result of analysis with the conventional shell shows that it cannot calculate the effect of coining that a bending moment decreases owing to pressure, the proposed element can express this effect similarly to a solid element. Therefore, this result shows that suggested shell element can treat normal stress similarly to a solid element, although the conventional shell element cannot calculate it. 


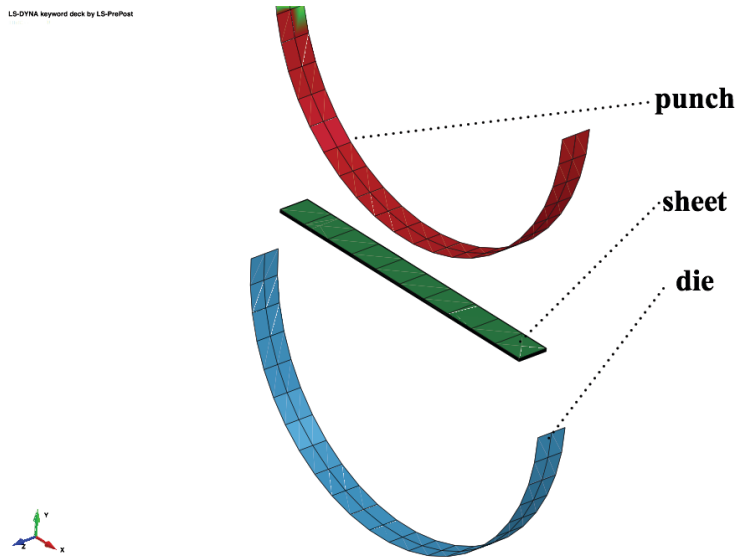

Figure 7. FE model used in coining simulation employing LSDYNA.

Table 2. Material properties.

\begin{tabular}{|c|c|c|c|}
\hline $\begin{array}{c}\text { Young's } \\
\text { modulus /GPa }\end{array}$ & $\begin{array}{c}\text { Poisson's } \\
\text { ratio }\end{array}$ & $\begin{array}{c}\text { Plastcity } \\
\text { coefficient } \\
\text { /MPa }\end{array}$ & $\begin{array}{c}\text { Work } \\
\text { hardening } \\
\text { coefficient }\end{array}$ \\
\hline 206 & 0.3 & 1300 & 0.45 \\
\hline
\end{tabular}

Table 3. Results of coining simulation.

\begin{tabular}{|c|c|c|c|}
\hline \multirow{2}{*}{$\begin{array}{c}\text { Punch } \\
\text { displacement } \\
\text { /mm }\end{array}$} & \multicolumn{3}{|c|}{ Radius of curvature /mm } \\
\cline { 2 - 4 } & $\begin{array}{c}\text { Proposed } \\
\text { shell }\end{array}$ & $\begin{array}{c}\text { Conventional } \\
\text { shell }\end{array}$ & Solid \\
\hline 92 & 83.05 & 83.05 & 83.33 \\
\hline 92.25 & 80.91 & & 80.90 \\
\hline 95 & 80.69 & 84.54 & 80.65 \\
\hline
\end{tabular}

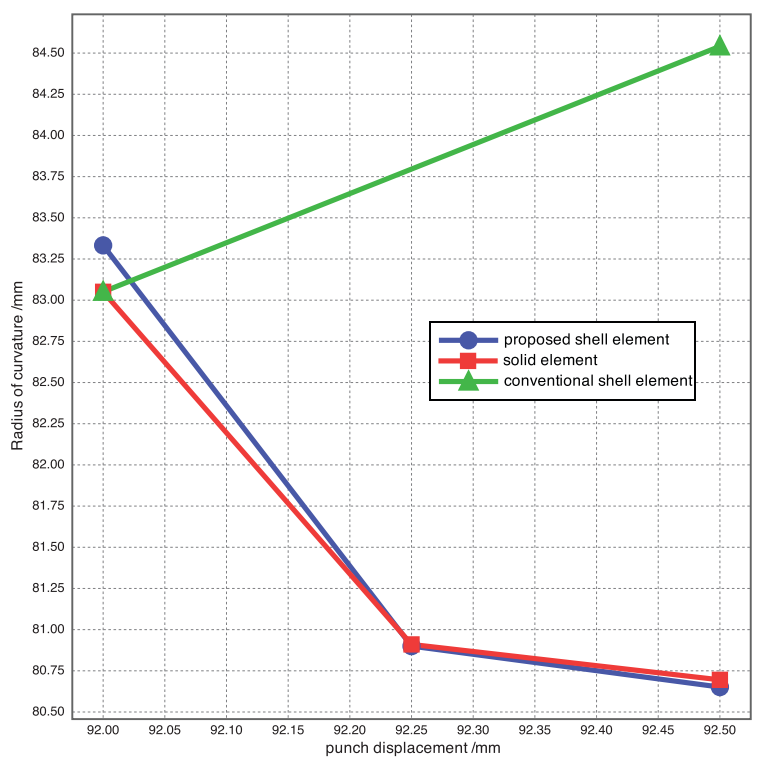

Figure 8. Results of coining simulation.

\section{Conclusion}

In this paper, new shell element with pseudo nodes for use in a mechanics-based springback compensation system was introduced. First, to test the basic function of the proposed element, the forming analysis of a beam with constant curvature was demonstrated. As a result, it was verified that this element behaves as desired by comparison with the theoretical value. Secondly, to show the effectiveness of the proposed element for calculating the normal stress in the coining process, a coining simulation of constant-curvature bending was demonstrated. It was verified that this element can calculate the effect by which the bending moment of a sheet decreases as it is subjected to pressure.

As future work, we intended to apply this element, to more complex examples such as a case including torsion. To more easily compensate for the springback using the proposed element, it may be necessary to integrate each computation.

\section{References}

1. W. Gan, R.H. Wagoner, Die design method for sheet springback, Int. J. Mech. Sci., 46, (2004), 10971113.

2. A.P. Karafillis, M.C. Boyce, Tooling Design in Sheet Metal Forming Using Springback Calculations, Int. J. Mech. Sci., 34, 2 (1992), 113-131.

3. A.P. Karafillis, M.C. Boyce, Tooling and Binder Design for Sheet Metal Forming Compensating Springback Error, Int. J. Mech. Tools Manu, 36, 4 (1996), 503-526.

4. X.A. Yang, F. Ruan, A die design method for springback compensation based on displacement adjustment, Int. J. Mech. Sci., 53, 5 (2011), 399-406.

5. H.S. Cheng, J. Cao, An accelerated springback compensation method, Int. J. Mech. Sci., 49, 3 (2007), 267-279.

6. R. Lingbeek, J. Huetink, S. Ohnimus, M. Petzoldt, J.Weiher, The development of a finite elements based on springback compensation tool for sheet metal product, J. Mater. Process Technol, 169, 1 (2005), 115 -125 .

7. N. Doke, and T. Oya, A Novel Springback Compensation Method Based on Finite Element Analysis and Springback Mechanics, MetalForming,14,(2012), 335338. 